\title{
THE BLACK HOLE-DARK MATTER HALO CONNECTION
}

\author{
Bassem M. Sabra ${ }^{1}$, Charbel Saliba ${ }^{2,5}$, Maya Abi AkL ${ }^{2,3,5}$, and Gilbert Chahine ${ }^{2,4,5}$ \\ ${ }^{1}$ Department of Physics \& Astronomy, Notre Dame University-Louaize, P.O. Box 72 Zouk Mikael, Zouk Mosbeh, Lebanon; bsabra@ndu.edu.lb \\ ${ }_{2}^{2}$ Department of Physics, Lebanese University II, Fanar, Lebanon \\ ${ }^{3}$ Texas A\&M University at Qatar Texas A\&M Engineering Building, Education City, Doha, Qatar \\ ${ }^{4}$ European Synchrotron ESRF, Grenoble, 38043, France \\ Received 2013 September 5; accepted 2015 January 30; published 2015 April 6
}

\begin{abstract}
We explore the connection between the central supermassive black holes (SMBH) in galaxies and the dark matter halo through the relation between the masses of the SMBHs and the maximum circular velocities of the host galaxies, as well as the relationship between stellar velocity dispersion of the spheroidal component and the circular velocity. Our assumption here is that the circular velocity is a proxy for the mass of the dark matter halo. We rely on a heterogeneous sample containing galaxies of all types. The only requirement is that the galaxy has a direct measurement of the mass of its SMBH and a direct measurement of its circular velocity and its velocity dispersion. Previous studies have analyzed the connection between the SMBH and dark matter halo through the relationship between the circular velocity and the bulge velocity dispersion, with the assumption that the bulge velocity dispersion stands in for the mass of the SMBH, via the well-established SMBH mass-bulge velocity dispersion relation. Using intermediate relations may be misleading when one is studying them to decipher the active ingredients of galaxy formation and evolution. We believe that our approach will provide a more direct probe of the SMBH and the dark matter halo connection. We find that the correlation between the mass of SMBHs and the circular velocities of the host galaxies is extremely weak, leading us to state the dark matter halo may not play a major role in regulating the black hole growth in the present Universe.
\end{abstract}

Key words: black hole physics - dark matter - galaxies: fundamental parameters - galaxies: halos

\section{INTRODUCTION}

Data has been accumulating over the last several years on an increasing number of galaxies in terms of measurements of the bulge velocity dispersion $\sigma$, mass of the central supermassive black hole $(\mathrm{SMBH}) M_{\mathrm{bh}}$, and the circular velocity, $v_{\mathrm{c}}$, of the host galaxy (Kronawitter et al. 2000; Palunas \& Williams 2000; Merritt \& Ferrarese 2001; Prugniel et al. 2001; Bernardi et al. 2002; Ferrarese 2002; Tremaine et al. 2002; Baes et al. 2003; Nelson et al. 2004; Onken et al. 2004; Peterson et al. 2004; Pizzella et al. 2005; Bedregal et al. 2006; Courteau et al. 2007; Ho 2007; Graham 2008; Hu 2008, 2009; McConnell \& Ma 2013). With the increase in the number of galaxies for which $\sigma, v_{\mathrm{c}}$, and $M_{\mathrm{bh}}$ have been measured, it is becoming possible to ask, and attempt to answer, questions about the processes that govern the formation of galaxies and their central SMBHs, and the symbiotic relationship between an SMBH and its host galaxy. One way to approach these issues is to compare observed correlations, or lack thereof, between $v_{\mathrm{c}}, \sigma$, and $M_{\mathrm{bh}}$ with theoretical predictions obtained from models of galaxy/SMBH formation (e.g., Haehnelt \& Kauffmann 2000; Kauffmann \& Haehnelt 2000; Di Matteo et al. 2003, 2008).

The observed $M_{\mathrm{bh}}-\sigma$ relation is now on a firm basis (Ferrarese \& Merritt 2000; Gebhardt et al. 2000; Tremaine et al. 2002; Graham 2008; Hu 2008, 2009; Gültenkin et al. 2009a; Beifiori et al. 2012) and it points to a common history between the SMBH and the spheroidal component of galaxies. Moreover, it was recently found that $v_{\mathrm{c}}$ and $\sigma$ are correlated (Ferrarese 2002; Baes et al. 2003; Courteau et al. 2007). However, Ho (2007) by a using a sample of 792 galaxies has

\footnotetext{
Former undergraduate students at the Department of Physics, Lebanese University II, Fanar, Lebanon.
}

shown that the $v_{\mathrm{c}}-\sigma$ relation has a very large scatter, and it depends on galaxy morphology.

Ferrares (2002) investigated the possibility of the masses of the SMBH being correlated with the total gravitational mass of the host galaxy or the mass of the dark matter halo. The author found a tight correlation between $v_{\mathrm{c}}$ and $\sigma$ for a sample of 36 galaxies, and by using the well-known $M_{\mathrm{bh}}-\sigma$ relation, and connecting the mass of the dark matter halo with the circular velocity of the galaxy, report a correlation between the mass of the SMBH and the circular velocity of the host galaxy. Baes et al. (2003) reported a relation between $M_{\mathrm{bh}}$ and $v_{\mathrm{c}}$. The rationale behind this correlation is that both quantities depend on the mass of the dark matter halo. A heavier halo will result in a deeper potential well that will give rise to a higher $M_{\mathrm{bh}}$, and at the same time lead to a higher $v_{\mathrm{c}}$. Zasov et al. (2005), on the other hand, found a very weak correlation between $M_{\mathrm{bh}}$ and $v_{\mathrm{c}}$. However, the $M_{\text {bh }}$ values in both Baes et al. (2003) and Zasov et al. (2005), as well as in Ferrarese (2002), were obtained from the $M_{\mathrm{bh}}-\sigma$ relation. Ho et al. (2008) report a correlation between $M_{\mathrm{bh}}$ and $v_{\mathrm{c}}$ for a sample of active galaxies, where the SMBH masses were measured using the virial method (Kaspi et al. 2000; Greene \& Ho 2005). More recently, Kormendy \& Bender (2011) used dynamical $M_{\mathrm{bh}}$ measurements to find that $M_{\mathrm{bh}}-v_{\mathrm{c}}$ relation is very weak in a sample of 25 spiral galaxies. The same result is confirmed in Beifiori et al. (2012) who used a large sample, 105 galaxies, but the used $M_{\mathrm{bh}} \mathrm{s}$ are a mix of dynamical measurements from Gultekin et al. (2009a) and upper limits from Beifiori et al. (2009), with the upper limits taken as surrogate of black mass. More recently, Sun (2013) analyzed the $M_{\mathrm{bh}}-v_{\mathrm{c}}$ relation for 22 galaxies with $v_{\mathrm{c}}$ determined from $\mathrm{H}_{\mathrm{I}}$ observations, and came up with a broad relation with large instrinsic scatter.

Given the controversy surrounding the $M_{\mathrm{bh}}-v_{\mathrm{c}}$ relation and its strength, using relations to find black holes masses will only 
introduce additional scatter and make it more difficult to reach firm conclusions for or against a genuine relation. Most of the previous studies that have investigated the relation between $M_{\mathrm{bh}}$ and the circular velocity did so indirectly since they "measured" the SMBH masses through the $M_{\mathrm{bh}}-\sigma$ relation for galaxies with measured $v_{\mathrm{c}}$ and $\sigma$. The danger in the indirect approach is that one is at the risk of masking, or leading to, a correlation between $M_{\mathrm{bh}}-v_{\mathrm{c}}$ through the $v_{\mathrm{c}}-\sigma$ and $M_{\mathrm{bh}}-\sigma$, and it necessarily ignores bulge less spirals with SMBHs, further masking the true connection between the SMBH and the dark matter halo.

The aim of this paper is to present a direct study of the nature of the $M_{\mathrm{bh}}-v_{\mathrm{c}}$ relation for 53 galaxies with dynamically measured $M_{\mathrm{bh}}$. This is the largest sample to date of galaxies that have these two properties available. We also present, as a by product of our study, the $M_{\mathrm{bh}}-\sigma$ relation based on 89 galaxies. We are revisiting the question of the $M_{\mathrm{bh}}-v_{\mathrm{c}}$ relation with a slightly "cleaner" sample of black hole masses. It is our hope here that by using dynamical $M_{\mathrm{bh}} \mathrm{s}$ we will be able to probe directly the intrinsic relations between a SMBH and the dark matter halo of the host galaxy.

\section{DESCRIPTION OF THE SAMPLE}

Our main objective is to build a sample of galaxies that have dynamical $M_{\mathrm{bh}}$ and $v_{\mathrm{c}}$ measurements. In order to make sure that we are not missing any objects we collected a large sample of 376 galaxies of all types for which measurements of $M_{\mathrm{bh}}, v_{\mathrm{c}}$, and/or $\sigma$ exist. This large sample was taken from papers that present measurements of SMBH masses (Richstone et al. 1998; Gebhardt et al. 2000; Kaspi et al. 2000; Nelson et al. 2004; Onken et al. 2004; Peterson et al. 2004; Ferrarese \& Ford 2005; de Fransesco et al. 2006; Pastorini et al. 2007; Gültenkin et al. 2009a; McConnell \& Ma 2013), papers that study the $M_{\mathrm{bh}}-\sigma$ relation (Ferrarese \& Merritt 2000; Gebhardt et al. 2000; Tremaine et al. 2002; Graham 2008; Hu 2008, 2009; Gultekin et al. 2009a), and papers that deal with the $v_{\mathrm{c}}-\sigma$ and/or $M_{\mathrm{bh}}-v_{\mathrm{c}}$ relationships (Ferrarese 2002; Baes et al. 2003; Pizzella et al. 2005; Courteau et al. 2007; Ho 2007; Kormendy \& Bender 2011).

We found 342 galaxies with $\sigma$ measurements, 269 galaxies with $v_{\mathrm{c}}$, and 125 galaxies with $M_{\mathrm{bh}}$. We reject 35 out of the 125 black hole masses since they are determined via reverberation mapping, which is calibrated to the $M_{\mathrm{bh}}-\sigma$ relation (Onken et al. 2004; Peterson et al. 2004). The intersections between these subsets resulted in 53 galaxies that have dynamical $M_{\mathrm{bh}}$ and $v_{\mathrm{c}}, 89$ galaxies with both dynamical $M_{\mathrm{bh}}$ and $\sigma$, and 251 galaxies that have both $\sigma$ and $v_{\mathrm{c}}$.

When error bars on a quantity for a particular galaxy are not available we assign an error by multiplying that quantity by the average percent error derived for that quantity from all galaxies that have errors. Out of 342 galaxies that have $\sigma$ values, 332 have errors listed, and 251 out of 269 galaxies have errors on $v_{\mathrm{c}}$. The average percent error on $\sigma$ is $\sim 10 \%$ and on $v_{\mathrm{c}}$ is $\sim 5 \%$. All available SMBH dynamical mass measurements have error bars quoted in the literature. The errors bars on $M_{\mathrm{bh}}$ are not symmetric. We calculate the average of the interval and assign this error to the data point when peforming the fits.

We list in Table 1 our final sample of 89 galaxies with dynamically-determined SMBH masses, stellar velocity dispersions, and circular velocities (53 out of 89). All of the $\sigma$ values are from HyperLeda ${ }^{1}$ (Paturel et al. 2003) except for IC
2560 (Garcia-Rissman et al. 2005) and Cygnus A (Graham 2008), and MW (Ferrarese 2002; Baes et al. 2003). Most of the black hole masses are from $\mathrm{Hu}$ (2009) and McConnell \& Ma (2013) except for NGC 1300, NGC 2748, NGC 2778, NGC 4342, NGC 4374, NGC 4945, NGC 7582 (Graham 2008), NGC 4303 (Pastorini et al. 2007), NGC 4486 Kormendy et al. (1996), NGC 4594 (Kormendy 1988). The distances in Table 1 are from the above mentioned papers. We include in Table 1 references to the original papers and the methods used to measure the black hole masses.

We use circular velocities from optical rotation curves whenever available: NGC 2787, NGC 1023, NGC 3115, NGC 3384, and NGC 4649 (Neistein et al. 1999), NGC 1399, NGC 3379, NGC 4374, NGC 4486, and NGC 4486 B (Kronawitter et al. 2000), and NGC 5846 (Pizzella et al. 2005). We use circular velocities derived from $\mathrm{H}_{\mathrm{I}}$ line widths as listed in Courteau et al. (2007) for: MW, NGC 224, and NGC 4258 (Ferrarese 2002); NGC 2974 (Pizzella et al. 2005); and NGC 3031, NGC 3227, NGC 4303, and NGC 4594 (Prugniel et al. 2001). We use at face value the circular velocities in HyperLeda for the remaining 34 galaxies. They are also based on $\mathrm{H}$ I line widths, which brings the number of galaxies in our sample with $v_{\mathrm{c}}$ derived from $\mathrm{H}$ I line widths to 42 . We note here that the results that we arrive at below do not depend on the origin of the circular velocities.

\section{ANALYSIS AND DISCUSSION}

Studying the correlations involves fitting straight lines in $\log -\log$ space: $\log y=\alpha+\beta \log x$. The results of the fitting have been known to depend in part on the details of the linear regression analysis (Tremaine et al. 2002). For the sake of definiteness, we limit ourselves to the prescription of Press et al. (1992) for data with symmetric error bars on both variables. The errors on $\alpha$ and $\beta$ are $1-\sigma$ fitting errors when $\chi^{2}$ differs by unity from its mininum value. A shortcoming of this approach is that it does not take into account the presence of intrinsic dispersion in the data. We follow the quick fix offered by Tremaine et al. (2002): we add in quadrature a parameter to the error of the $y$-coordinates (Gebhardt et al. 2000). This parameter is a measure of the intrinsic dispersion. Its value is adjusted by hand to lead a reduced chi squared of unity. This prescription (Tremaine et al. 2002) is essentially implemented in FITEXY and, its more advanced variant MPFITEXY (Markwardt 2009; Williams et al. 2010), which is what we use in this paper.

\subsection{A $M_{\mathrm{bh}}-v_{c}$ Relation?}

We plot in Figure $1 \log \left(M_{\mathrm{bh}} / 10^{8} M_{\odot}\right)$ against $\log \left(v_{\mathrm{c}} / 200 \mathrm{~km} \mathrm{~s}^{-1}\right)$ for 53 galaxies, made up of 13 ellipticals, 16 lenticulars, and 24 spirals (Table 1). It is clear that three galaxies whose $v_{\mathrm{c}} \lesssim 100 \mathrm{~km} \mathrm{~s}^{-1}$ do not fall in the region where the other data points cluster. These 3 galaxies are IC 1459 (E3), NGC 5252 (S0), and NGC 3608 (E2). We do not include them in our subsequent analysis when we fit:

$$
\log \frac{M_{\mathrm{bh}}}{10^{8} M_{\odot}}=\alpha+\beta \log \frac{v_{\mathrm{c}}}{200 \mathrm{~km} \mathrm{~s}^{-1}}
$$

to the data points in Figure 1.

A casual inspection of Figure 1 shows that the correlation between the $M_{\mathrm{bh}}$ and $v_{\mathrm{c}}$, if there is any, is very weak. Taken 
Table 1

Galaxy Sample

\begin{tabular}{|c|c|c|c|c|c|c|c|}
\hline $\begin{array}{l}\text { Galaxy } \\
\text { (1) }\end{array}$ & $\begin{array}{c}\text { MORPH } \\
(2)\end{array}$ & $\begin{array}{c}\mathrm{D}(\mathrm{Mpc}) \\
(3)\end{array}$ & $\begin{array}{l}\text { A } \\
(4)\end{array}$ & $\begin{array}{c}M_{\mathrm{bh}} /\left(10^{6} M_{\odot}\right) \\
(5)\end{array}$ & $\begin{array}{c}v_{c}\left(\mathrm{~km} \mathrm{~s}^{-1}\right) \\
(6)\end{array}$ & $\begin{array}{c}\sigma\left(\mathrm{km} \mathrm{s}^{-1}\right) \\
(7)\end{array}$ & $\begin{array}{c}\operatorname{Rf}\left(M_{\mathrm{bh}}\right) \\
(8)\end{array}$ \\
\hline$\overline{\text { Circinus }}$ & SB & 2.8 & $\mathrm{Y}$ & $1.10_{-0.23}^{+0.18}$ & $158.22 \pm 6.04$ & $157.63 \pm 18.77$ & $1 \mathrm{~m}$ \\
\hline IC 1459 & E3 & 29.2 & $\mathrm{Y}$ & $2511.89_{-462.71}^{+462.71}$ & $35.87 \pm 2.86$ & $306.1 \pm 7.8$ & $2 \mathrm{~s}$ \\
\hline IC 2560 & $\mathrm{SBb}, \mathrm{p}$ & 41.4 & $\mathrm{Y}$ & $2.88_{-0.66}^{+0.53}$ & $196.08 \pm 3.12$ & $137 \pm 14$ & $3 \mathrm{~m}$ \\
\hline MW & $\mathrm{SBbc}$ & 0.008 & $\mathrm{Y}$ & $4.10_{-0.6}^{+0.6}$ & $180 \pm 20$ & $100 \pm 20$ & $4 p$ \\
\hline NGC 0224 & $\mathrm{Sb}$ & 0.76 & $\mathrm{Y}$ & $141.25_{-32.52}^{+71.55}$ & $249.84 \pm 6.73$ & $169.87 \pm 5.12$ & $5 \mathrm{~s}$ \\
\hline NGC 1023 & SB0 & 11.4 & $\mathrm{~N}$ & $43.65_{-5.03}^{+5.03}$ & $250 \pm 17$ & $204.48 \pm 4.23$ & $6 s$ \\
\hline NGC 1068 & $\mathrm{Sb}$ & 15.3 & $\mathrm{Y}$ & $8.32_{-0.38}^{+0.38}$ & $321.01 \pm 21.98$ & $198.68 \pm 17.02$ & $7 \mathrm{~m}$ \\
\hline NGC 1194 & So & 55.5 & $\mathrm{Y}$ & $68.00_{-3}^{+3}$ & $202.84 \pm 16.27$ & $147.9 \pm 23.95$ & $47 \mathrm{~m}$ \\
\hline NGC 1300 & $\mathrm{SBbc}$ & 20.7 & $\mathrm{~N}$ & $73.00_{-35}^{+69}$ & $167.09 \pm 4.37$ & $185.9 \pm 46.59$ & $8 \mathrm{~g}$ \\
\hline NGC 1332 & So & 22.7 & $\mathrm{~N}$ & $1500.00_{-200}^{+200}$ & $138.12 \pm 7.77$ & $328 \pm 16$ & $49 \mathrm{~s}$ \\
\hline NGC 1399 & $\mathrm{E}$ & 21.1 & $\mathrm{~N}$ & $1202.26_{-830.49}^{+415.25}$ & $424 \pm 46$ & $341.88 \pm 5.84$ & $9 \mathrm{~s}$ \\
\hline NGC 2273 & $\mathrm{SBa}$ & 26.8 & $\mathrm{Y}$ & $7.80_{-0.4}^{+0.4}$ & $192.09 \pm 5.46$ & $144.5 \pm 16.7$ & $47 \mathrm{~m}$ \\
\hline NGC 2748 & $\mathrm{Sc}$ & 24.9 & $\mathrm{~N}$ & $47.00_{-38.4}^{+38}$ & $150 \pm 10$ & $81 \pm 1$ & $8 \mathrm{~g}$ \\
\hline NGC 2787 & $\mathrm{SB} 0 / \mathrm{a}$ & 7.5 & $\mathrm{Y}$ & $40.74_{-5.63}^{+3.75}$ & $181.77 \pm 13.5$ & $193.61 \pm 6.29$ & $10 \mathrm{~g}$ \\
\hline NGC 2960 & $\mathrm{Sa}$ & 75.3 & $\mathrm{Y}$ & $12.10_{-0.5}^{+0.5}$ & $300.62 \pm 13.41$ & $166 \pm 15.35$ & $47 \mathrm{~m}$ \\
\hline NGC 2974 & $\mathrm{E} 4$ & 21.5 & $\mathrm{Y}$ & $169.82_{-19.55}^{+19.55}$ & $105.22 \pm 5.67$ & $238.23 \pm 4.21$ & $11 \mathrm{~s}$ \\
\hline NGC 3031 & $\mathrm{Sb}$ & 3.9 & $\mathrm{Y}$ & $79.43_{-10.97}^{+18.29}$ & $224.51 \pm 9.38$ & $161.61 \pm 3.1$ & $12 \mathrm{~g}$ \\
\hline NGC 3079 & $\mathrm{SB}(\mathrm{s}) \mathrm{c}$ & 19.1 & $\mathrm{Y}$ & $2.51_{-1.74}^{+1.74}$ & $210.19 \pm 5.15$ & $145.66 \pm 9.71$ & $13 \mathrm{~m}$ \\
\hline NGC 3115 & So & 9.7 & $\mathrm{~N}$ & $933.25_{-322.33}^{+429.78}$ & $369 \pm 61$ & $267.6 \pm 4.13$ & $14 \mathrm{~s}$ \\
\hline NGC 3227 & $\mathrm{SBa}$ & 17.5 & $\mathrm{Y}$ & $19.95_{-21.13}^{+10.57}$ & $130.05 \pm 4.1$ & $134.56 \pm 5.71$ & $15 \mathrm{~g}, \mathrm{~s}$ \\
\hline NGC 3245 & So & 20.9 & $\mathrm{~N}$ & $208.93_{-57.73}^{+43.3}$ & $290 \pm 5$ & $209.91 \pm 8.36$ & $40 \mathrm{~g}$ \\
\hline NGC 3379 & E1 & 10.3 & $\mathrm{~N}$ & $120.23_{-83.05}^{+49.83}$ & $259 \pm 23$ & $209.23 \pm 2.1$ & $16 \mathrm{~g}, \mathrm{~s}$ \\
\hline NGC 3384 & So & 11.6 & $\mathrm{~N}$ & $17.38_{-2.4}^{+1.2}$ & $245 \pm 30$ & $148.35 \pm 3.4$ & $17 \mathrm{~s}$ \\
\hline NGC 3393 & Sba,p & 51.8 & $\mathrm{Y}$ & $30.90_{-2.13}^{+2.13}$ & $157.93 \pm 10.61$ & $197.14 \pm 28.35$ & $18 \mathrm{~m}$ \\
\hline NGC 3414 & SB0 & 25.2 & $\mathrm{~N}$ & $251.19_{-34.7}^{+28.92}$ & $139.02 \pm 7.26$ & $236.75 \pm 7.47$ & $11 \mathrm{~s}$ \\
\hline NGC 3585 & So & 21.2 & $\mathrm{~N}$ & $338.84_{-62.42}^{+124.83}$ & $280 \pm 20$ & $205.67 \pm 6.83$ & $30 \mathrm{~s}$ \\
\hline NGC 3608 & E2 & 22.9 & $\mathrm{~N}$ & $208.93_{-76.97}^{+86.59}$ & $85.36 \pm 7.15$ & $192.08 \pm 3.4$ & $17 \mathrm{~s}$ \\
\hline NGC 3998 & So & 18.3 & $\mathrm{Y}$ & $288.40_{-53.13}^{+26.56}$ & $407.61 \pm 41.79$ & $279.82 \pm 14.74$ & $19 \mathrm{~g}$ \\
\hline NGC 4026 & So & 15.6 & $\mathrm{~N}$ & $208.93_{-43.3}^{+57.73}$ & $255 \pm 10$ & $177.98 \pm 4.45$ & $30 \mathrm{~s}$ \\
\hline NGC 4151 & SAB & 13.9 & $\mathrm{Y}$ & $32.36_{-40.98}^{+7.45}$ & $144.27 \pm 9.54$ & $103.22 \pm 9.91$ & $20 \mathrm{~g}, \mathrm{~s}$ \\
\hline NGC 4258 & $\mathrm{SABbc}$ & 7.2 & $\mathrm{Y}$ & $38.90_{-0.9}^{+0.9}$ & $208.09 \pm 6.17$ & $134.4 \pm 17.18$ & $21 \mathrm{~m}$ \\
\hline NGC 4303 & $\mathrm{SBbc}$ & 16.1 & $\mathrm{Y}$ & $5.00_{-2.26}^{+0.87}$ & $213.78 \pm 7.25$ & $108.72 \pm 11.66$ & $22 \mathrm{~g}$ \\
\hline NGC 4342 & So & 17 & $\mathrm{~N}$ & $330.00_{-110}^{+190}$ & $311 \pm 10$ & $252.07 \pm 8.39$ & $42 \mathrm{~s}$ \\
\hline NGC 4374 & $\mathrm{E}$ & 18.4 & $\mathrm{Y}$ & $464.00_{-183}^{+346}$ & $410 \pm 30$ & $283.29 \pm 2.81$ & $23 \mathrm{~g}$ \\
\hline NGC 4388 & SB & 19.8 & $\mathrm{Y}$ & $8.80_{-0.2}^{+0.2}$ & $171.34 \pm 8.57$ & $107.2 \pm 7.4$ & $47 \mathrm{~m}$ \\
\hline NGC 4486 & $\mathrm{E}$ & 17.2 & $\mathrm{Y}$ & $3630.78_{-1170.43}^{+919.62}$ & $507 \pm 38$ & $334.44 \pm 5.05$ & $24 \mathrm{~g}$ \\
\hline NGC 4486B & $\mathrm{E}$ & 15.3 & $\mathrm{~N}$ & $570.00_{-269.00}^{+322.00}$ & $249 \pm 17$ & $169.93 \pm 3.94$ & $25 \mathrm{~s}$ \\
\hline NGC 4526 & So & 16.5 & $\mathrm{~N}$ & $473.00_{-13}^{+13}$ & $150.41 \pm 7.52$ & $222 \pm 11$ & $50 \mathrm{~g}$ \\
\hline NGC 4564 & E3 & 15.9 & $\mathrm{~N}$ & $58.88_{-8.14}^{+2.71}$ & $229 \pm 2$ & $157.4 \pm 3.1$ & $17 \mathrm{~s}$ \\
\hline NGC 4594 & $\mathrm{Sa}$ & 9.8 & $\mathrm{Y}$ & $1000.00_{-700.00}^{+1000.00}$ & $358.48 \pm 10.32$ & $241.65 \pm 4.41$ & $26 s$ \\
\hline NGC 4596 & SB0 & 16.7 & $\mathrm{~N}$ & $77.62_{-42.9}^{+33.96}$ & $154.85 \pm 7.88$ & $148.8 \pm 2.85$ & $10 \mathrm{~g}$ \\
\hline NGC 4649 & E1 & 17.3 & $\mathrm{~N}$ & $1995.26_{-689.14}^{+367.54}$ & $378 \pm 99$ & $335.3 \pm 4.45$ & $17 \mathrm{~s}$ \\
\hline NGC 4736 & $\mathrm{Sab}$ & 4.9 & $\mathrm{Y}$ & $6.68_{-1.54}^{+1.54}$ & $181 \pm 10$ & $112 \pm 3$ & $52 \mathrm{~s}$ \\
\hline NGC 4826 & $\mathrm{Sab}$ & 6.4 & $\mathrm{Y}$ & $1.36_{-0.34}^{+0.35}$ & $155 \pm 5$ & $91.47 \pm 4.27$ & $52 \mathrm{~s}$ \\
\hline NGC 4945 & SB & 3.8 & $\mathrm{Y}$ & $1.40_{-0.7}^{+1.4}$ & $167.05 \pm 9$ & $127.92 \pm 19.09$ & $27 \mathrm{~m}$ \\
\hline NGC 5128 & S0 & 3.5 & $\mathrm{Y}$ & $50.12_{-5.77}^{+4.62}$ & $348.26 \pm 15.44$ & $119.77 \pm 7.13$ & $28 \mathrm{~g}$ \\
\hline NGC 5252 & So & 96.8 & $\mathrm{Y}$ & $1000.00_{-690.78}^{+921.03}$ & $57.6 \pm 3.44$ & $196.46 \pm 27.92$ & $29 \mathrm{~g}$ \\
\hline NGC 5576 & E3 & 27.1 & $\mathrm{~N}$ & $181.97_{-46.09}^{+29.33}$ & $103.4 \pm 5.57$ & $170.68 \pm 4.82$ & $30 \mathrm{~s}$ \\
\hline NGC 5846 & E0 & 24.9 & $\mathrm{~N}$ & $1096.48_{-100.99}^{+100.99}$ & $421.24 \pm 19$ & $239.03 \pm 4.14$ & $11 \mathrm{~s}$ \\
\hline NGC 7052 & $\mathrm{E} 4$ & 71.4 & $\mathrm{~N}$ & $398.11_{-201.67}^{+210.84}$ & $150.35 \pm 8.1$ & $284.42 \pm 13.22$ & $31 \mathrm{~g}$ \\
\hline NGC 7457 & So & 13.2 & $\mathrm{~N}$ & $3.80_{-1.4}^{+1.05}$ & $105 \pm 5$ & $68.85 \pm 4.04$ & $17 \mathrm{~s}$ \\
\hline NGC 7582 & SBab & 22 & $\mathrm{Y}$ & $55.00_{-19}^{+26}$ & $194.99 \pm 3.32$ & $156.46 \pm 19.93$ & $32 \mathrm{~g}$ \\
\hline UGC 3789 & $\mathrm{Sa}$ & 48.4 & $\mathrm{~N}$ & $10.80_{-0.5}^{+0.6}$ & $273.4 \pm 19.78$ & $107.2 \pm 12.35$ & $47 \mathrm{~m}$ \\
\hline
\end{tabular}

Galaxies with no $v_{\mathrm{c}}$

\begin{tabular}{lllllllll}
\hline A1836 & E & 157.5 & N & $3900.00_{-600}^{+400}$ & $\ldots$ & $288 \pm 14$
\end{tabular}


Table 1

(Continued)

\begin{tabular}{|c|c|c|c|c|c|c|c|}
\hline $\begin{array}{l}\text { Galaxy } \\
\text { (1) }\end{array}$ & $\begin{array}{c}\text { MORPH } \\
\text { (2) }\end{array}$ & $\begin{array}{c}\mathrm{D}(\mathrm{Mpc}) \\
(3)\end{array}$ & $\begin{array}{l}\text { A } \\
(4)\end{array}$ & $\begin{array}{c}M_{\mathrm{bh}} /\left(10^{6} M_{\odot}\right) \\
(5)\end{array}$ & $\begin{array}{c}v_{\mathrm{c}}\left(\mathrm{km} \mathrm{s}^{-1}\right) \\
(6)\end{array}$ & $\begin{array}{c}\sigma\left(\mathrm{km} \mathrm{s}^{-1}\right) \\
(7)\end{array}$ & $\begin{array}{c}\operatorname{Rf}\left(M_{\mathrm{bh}}\right) \\
\quad(8)\end{array}$ \\
\hline A3565 & $\mathrm{E}$ & 54.4 & $\mathrm{~N}$ & $1400.00_{-200}^{+300}$ & $\ldots$ & $322 \pm 16$ & $34 \mathrm{~g}$ \\
\hline Cygnus A & E & 240 & $\mathrm{Y}$ & $2884.03_{-796.89}^{+597.67}$ & $\ldots$ & $270 \pm 27.44$ & $33 \mathrm{~g}$ \\
\hline IC 4296 & $\mathrm{cD}$ & 50.8 & $\mathrm{Y}$ & $1348.96_{-217.43}^{+186.37}$ & $\ldots$ & $333.24 \pm 5.87$ & $34 \mathrm{~s}, \mathrm{~g}$ \\
\hline NGC 0221 & E2 & 0.81 & $\mathrm{~N}$ & $2.51_{-0.06}^{+0.46}$ & $\ldots$ & $72.21 \pm 1.96$ & $35 \mathrm{~s}$ \\
\hline NGC 0524 & SO,c & 23.3 & $\mathrm{~N}$ & $831.76_{-38.3}^{+57.46}$ & $\ldots$ & $253.46 \pm 7.78$ & $36 \mathrm{~s}$ \\
\hline NGC 0821 & E4 & 24.1 & $\mathrm{~N}$ & $85.11_{-45.08}^{+29.4}$ & $\ldots$ & $200.01 \pm 3.21$ & $37 \mathrm{~s}$ \\
\hline NGC 1277 & so & 73 & $\mathrm{~N}$ & $17000.00_{-3000}^{+3000}$ & $\ldots$ & $333 \pm 17$ & $48 \mathrm{~s}$ \\
\hline NGC 1316 & SAB & 20 & $\mathrm{~N}$ & $162.18_{-29.87}^{+26.14}$ & $\ldots$ & $225.85 \pm 3.36$ & $38 \mathrm{~s}$ \\
\hline NGC 1407 & $\mathrm{E}$ & 29 & $\mathrm{~N}$ & $4700.00_{-500}^{+900}$ & $\ldots$ & $274 \pm 14$ & $49 \mathrm{~s}$ \\
\hline NGC 1550 & $\mathrm{E}$ & 53 & $\mathrm{~N}$ & $3800.00_{-400}^{+400}$ & $\ldots$ & $289 \pm 14$ & $49 s$ \\
\hline NGC 2549 & So & 12.3 & $\mathrm{~N}$ & $14.13_{-21.79}^{+0.98}$ & $\ldots$ & $143.66 \pm 3.52$ & $36 \mathrm{~s}$ \\
\hline NGC 2778 & E2 & 22.9 & $\mathrm{~N}$ & $14.00_{-9}^{+8}$ & $\ldots$ & $161.67 \pm 3.16$ & $17 \mathrm{~s}$ \\
\hline NGC 3091 & E & 52.7 & $\mathrm{~N}$ & $3700.00_{-200}^{+100}$ & $\ldots$ & $307 \pm 15$ & $49 \mathrm{~s}$ \\
\hline NGC 3377 & E5 & 11.2 & $\mathrm{~N}$ & $109.65_{-10.1}^{+75.74}$ & $\ldots$ & $138.72 \pm 2.57$ & $39 \mathrm{~s}$ \\
\hline NGC 3607 & SA & 19.9 & $\mathrm{Y}$ & $120.23_{-49.83}^{+33.22}$ & $\ldots$ & $223.5 \pm 9.8$ & $30 \mathrm{~s}$ \\
\hline NGC 4261 & E2 & 31.6 & $\mathrm{Y}$ & $524.81_{-120.84}^{+96.67}$ & $\ldots$ & $308.95 \pm 5.63$ & $41 \mathrm{~g}$ \\
\hline NGC 4291 & E2 & 26.2 & $\mathrm{~N}$ & $338.84_{-460.33}^{+78.02}$ & $\ldots$ & $285.3 \pm 5.69$ & $17 \mathrm{~s}$ \\
\hline NGC 4459 & So & 16.1 & $\mathrm{~N}$ & $70.79_{-14.67}^{+11.41}$ & $\ldots$ & $169.95 \pm 7.06$ & $10 \mathrm{~g}$ \\
\hline NGC 4473 & E5 & 15.3 & $\mathrm{~N}$ & $120.23_{-155.03}^{+35.99}$ & $\ldots$ & $179.25 \pm 2.96$ & $17 \mathrm{~s}$ \\
\hline NGC 4486A & E2 & 18.3 & $\mathrm{~N}$ & $\begin{array}{l}12.88_{-10.09}^{+3.56} \\
\text { (1) }\end{array}$ & $\ldots$ & $42.61 \pm 2.58$ & $43 \mathrm{~s}$ \\
\hline NGC 4552 & E & 15.9 & $\mathrm{Y}$ & $501.19_{-57.7}^{+46.16}$ & $\ldots$ & $252.65 \pm 3.28$ & $11 \mathrm{~s}$ \\
\hline NGC 4621 & E5 & 18.3 & $\mathrm{~N}$ & $398.11_{-45.83}^{+36.67}$ & $\ldots$ & $225.15 \pm 3.23$ & $11 \mathrm{~s}$ \\
\hline NGC 4697 & E4 & 11.7 & $\mathrm{~N}$ & $169.82_{-31.28}^{+19.55}$ & $\ldots$ & $170.92 \pm 1.95$ & $17 \mathrm{~s}$ \\
\hline NGC 4742 & E4 & 15.5 & $\mathrm{~N}$ & $14.13_{-6.18}^{+3.58}$ & $\ldots$ & $108.32 \pm 3.99$ & $44 \mathrm{~s}$ \\
\hline NGC 4751 & $\mathrm{E} / \mathrm{S} 0$ & 27.7 & $\mathrm{~N}$ & $1400.00_{-100}^{+100}$ & $\ldots$ & $355 \pm 18$ & $49 \mathrm{~s}$ \\
\hline NGC 5077 & $\mathrm{E} 3, \mathrm{c}$ & 40.2 & $\mathrm{Y}$ & $724.44_{-383.66}^{+350.3}$ & $\ldots$ & $255.87 \pm 7.54$ & $45 \mathrm{~g}$ \\
\hline NGC 5328 & $\mathrm{E}$ & 65.9 & $\mathrm{~N}$ & $4800.00_{-1900}^{+1000}$ & $\ldots$ & $333 \pm 17$ & $49 \mathrm{~s}$ \\
\hline NGC 5516 & $\mathrm{E}$ & 60.1 & $\mathrm{~N}$ & $3500.00_{-400}^{+200}$ & $\ldots$ & $306 \pm 26$ & $49 \mathrm{~s}$ \\
\hline NGC 5813 & E1 & 32.2 & $\mathrm{~N}$ & $707.95_{-81.51}^{+65.2}$ & $\ldots$ & $236.74 \pm 3.35$ & $11 \mathrm{~s}$ \\
\hline NGC 5845 & E3 & 25.9 & $\mathrm{~N}$ & $263.03_{-230.14}^{+42.39}$ & $\ldots$ & $237.61 \pm 9.17$ & $17 \mathrm{~s}$ \\
\hline NGC 6086 & E & 139.1 & $\mathrm{~N}$ & $3800.00_{-1200}^{+1700}$ & $\ldots$ & $318 \pm 16$ & $51 \mathrm{~s}$ \\
\hline NGC 6251 & E2 & 107 & $\mathrm{Y}$ & $616.60_{-255.56}^{+170.37}$ & $\ldots$ & $324.57 \pm 15.4$ & $46 \mathrm{~g}$ \\
\hline NGC 6264 & S & 145.4 & $\mathrm{Y}$ & $30.30_{-0.4}^{+0.5}$ & $\ldots$ & $158.5 \pm 14.65$ & $47 \mathrm{~m}$ \\
\hline NGC 6323 & $\mathrm{Sab}$ & 110.5 & $\mathrm{~N}$ & $9.80_{-0.1}^{+0.1}$ & $\ldots$ & $158.5 \pm 25.65$ & $47 \mathrm{~m}$ \\
\hline NGC 6861 & E/S0 & 28.9 & $\mathrm{~N}$ & $2100.00_{-200}^{+200}$ & $\ldots$ & $389 \pm 19$ & $49 \mathrm{~s}$ \\
\hline
\end{tabular}

Note. Col. (2): Morphological classification (NASA/IPAC Extragalactic Database. Col. (3): Distances (see col. (5) for references). Col. (4): Active Galaxy. Col. (5): black hole masses from Hu (2009), McConnell \& Ma (2013) except for NGC 1300, NGC 2778; NGC 4342; NGC 4374; and NGC 4945 (Graham 2008 ), NGC 4303 (Pastorini et al. 2007), NGC 4486 Kormendy et al. (1996), and NGC 4594 (Kormendy 1988). (8). Col. (6): Galaxy circular velocity (see text for references). Col. (7): Central velocity dispersion from HyperLeda. Col. (8): Original references for black holes masses. Methods for derivation of black hole masses in original references: $\mathrm{g}=$ gas dynamics; $\mathrm{s}=$ star dynamics; $\mathrm{p}=$ stellar proper motion; $\mathrm{m}=$ maser. Original references for black hole masses: (1) Greenhill (2003), (2) Cappellari et al. (2002), (3) Tilak et al. (2008), (4) Ghez et al. (2008), (5) Bender et al. (2005), (6) Bower et al. (2001), (7) Lodato et al. (2003), (8) Atkinson et al. (2005), (9) Houghton et al. (2006), (10) Sarzi et al. (2001), (11) Cappellari et al. (2007), (12) Devereux et al. (2003), (13) Yamauchi et al. (2004), (14) Kormendy et al. (1996), (15) Davis et al. (2006) and Hicks \& Malkan (2008), (16) Gebhardt et al. (2001) and Shapiro et al. (2006), (17) Gebhardt et al. (2003), (18) Kondratko et al. (2008), (19) de Francesco et al. (2006), (20) Onken et al. (2007) and Hicks \& Malkan (2008), (21) Hernstein et al. (1999), (22) Pastorini et al. (2007), (23) Maciejewski \& Binney (2001), (24) Harms et al. (1994) and Macchetto et al. (1997), (25) Kormendy et al. (1996), (26) Kormendy (1988), (27) Greenhill et al. (1997), (28) Neumayer et al. (2007), (29) Capetti et al. (2005), (30) Gultekin et al. (2009b), (31) van der Marel \& van der Bosch (1998), (32) Wold et al. (2006), (33) Tadhunter et al. (2003), (34) Dalla Bonta et al. (2009), (35) Verolme et al. (2002), (36) Krajnovic et al. (2009), (37) Richstone et al. (2004), (38) Nowak (2008), (39) Copin et al. (2004), (40) Barth et al. (2001), (41) Ferrarese et al. (1998), (43) Nowak (2007), (42) Cretton \& van der Bosch (1999) and Valluri et al. (2004), (44) Tremaine et al. (2002), (45) de Fransesco \& Capetti (2008), (46) Ferrarese \& Holland (1999), (47) Kuo et al. (2011), (48) van den Bosch et al. (2012), (49) Rusli et al. (2011), (50) Davies et al. (2013) and Gould (2013), (51) McConnell et al. (2011), (52) Kormendy \& Bender (2011).

separately, galaxies belonging to the same morphological type form essentially scatter plots and at best barely show a hint of a correlation. A visual inspection in Figure 1 that does not distinguish between the galaxy types can be misleading. An apparent correlation will appear to be present if one is not careful. The spirals, represented as blue crosses, have on average lower black hole masses and velocities than the ellipticals and the lenticulars. Placed on the same plot, this offset between spiral and E/S0 galaxies leads one to believe that there is a correlation. 


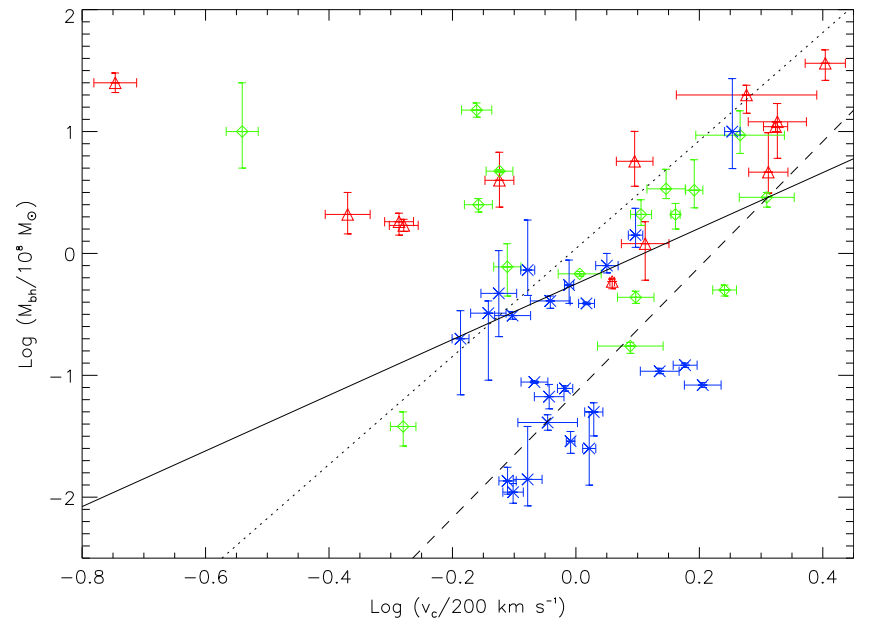

Figure 1. $\log \left(M_{\mathrm{bh}} / 10^{8} M_{\odot}\right)$ vs. $\log \left(v_{\mathrm{c}} / 200 \mathrm{~km} \mathrm{~s}^{-1}\right)$ for the 53 galaxies in Table 1 that have dynamical black hole mass and host galaxy circular velocity measurments. The ellipticals are the red triangles, the lenticulars are the green rhombii, and the spirals are the blue crosses. The solid line is the result of the fit, Equation (2). The three galaxies with $v_{\mathrm{c}} \lesssim 100 \mathrm{~km} \mathrm{~s}^{-1}$ are not included in the fit. The dashed line is the semi-observational $M_{\mathrm{bh}}-v_{\mathrm{c}}$ relation Equation (5), and the dotted line is Equation (7), a theoretical $M_{\mathrm{bh}}-v_{\mathrm{c}}$ relation.

To be more quantitative, we use the procedure outlined above to obtain:

$$
\begin{aligned}
\log \frac{M_{\mathrm{bh}}}{10^{8} M_{\odot}}= & (-0.25 \pm 0.11) \\
& +(2.28 \pm 0.67) \log \frac{v_{\mathrm{c}}}{200 \mathrm{~km} \mathrm{~s}^{-1}},
\end{aligned}
$$

with an intrinsic scatter of $0.75 \mathrm{dex}$. The intrinsic scatter is very large and so is the error on the slope. This leads us to conclude that there is no correlation between the mass of SMBH and the circular velocity of the host galaxy. This result is broadly consistent with those of Sun (2013), Beifiori et al. (2012), and Kormendy \& Bender (2011).

Previous authors have studied the relation between $M_{\mathrm{bh}}$ and $v_{\mathrm{c}}$ and their conclusions are varied. Baes et al. (2003) reported a slope of $4.21 \pm 0.60$ for a sample of 40 galaxies. However, Baes et al. (2003) determined the masses of the black holes indirectly by using the well-established $M_{\mathrm{bh}}-\sigma$ relation of Tremaine et al. (2002). They combined their $v_{\mathrm{c}}-\sigma$ relation with the $M_{\mathrm{bh}}-\sigma$ relation of Tremaine et al. (2002) to derive a $M_{\mathrm{bh}}-v_{\mathrm{c}}$ relation. Ho (2007), using a sample of 792 galaxies, has shown that the $v_{\mathrm{c}}-\sigma$ relation has appreciable variation depending on host galaxy properties. Ho (2007), cautions against replacing the bulge, i.e., stellar velocity $\sigma$, with the halo, $v_{\mathrm{c}}$, in attempts to use $v_{\mathrm{c}}$ to derive black hole masses based on a $M_{\mathrm{bh}}-v_{\mathrm{c}}$ relation. The slope arrived at by Baes et al. (2003) is in agreement with the predictions of cosmological simulations ( $\mathrm{Di}$ Matteo et al. 2003), but it is an artefact of combining the $M_{\mathrm{bh}}-\sigma$ relation with the less accurate $v_{\mathrm{c}}-\sigma$ relation. Zasov et al. (2005) found significant scatter in the $M_{\mathrm{bh}}-v_{\mathrm{c}}$ plots and concluded that the relation between $M_{\mathrm{bh}}$ and $v_{\mathrm{c}}$ is very weak. Their sample of 41 galaxies suffered contamination from galaxies, 20 in number, whose black hole masses were determined indirectly from the $M_{\mathrm{bh}}-\sigma$ relation. Sabra et al. (2008) reported a slope of $6.75 \pm 0.8$ based on a small sample of only 16 galaxies.

Bandara et al. (2009) reported a correlation between $M_{\mathrm{bh}}$ and $M_{\text {tot }}$, the total gravitational mass of the host galaxy. The total mass was determined from gravitational lensing observations, and their black hole masses were determined indirectly through the $M_{\mathrm{bh}}-\sigma$ relation (Gültenkin et al. 2009a). However, when they calculate $M_{\mathrm{bh}}$ through the $M_{\mathrm{bh}}-n$ relation (Graham et al. 2001, Graham \& Driver 2007), where $n$ is the Sérsic index, they find no signficant correlation between $M_{\mathrm{bh}}$ and $M_{\mathrm{tot}}$.

We derive here the expected $M_{\mathrm{bh}}-v_{\mathrm{c}}$ relation from the Bandara et al. (2009) $M_{\mathrm{bh}}-M_{\mathrm{tot}}$ relation:

$$
\begin{aligned}
\log M_{\mathrm{bh}}= & (8.18 \pm 0.11) \\
& +(1.55 \pm 0.31)\left(\log M_{\mathrm{tot}}-13\right) .
\end{aligned}
$$

We use Equation (5) in Ferrarse (2002), in which the author assumes that $v_{\mathrm{c}}$ is equal to the virial velocity and uses relations from Bullock et al. (2001) between the mass of the dark matter halo and the circular velocity. We further assume that the total gravitational mass is equal to the mass of the dark matter halo to get:

$$
\log \frac{M_{\mathrm{DM}}}{10^{12} M_{\odot}}=0.15+3.32 \log \frac{v_{\mathrm{c}}}{200 \mathrm{~km} \mathrm{~s}^{-1}} .
$$

Combining Equations (3) and (4) above we obtain the following:

$$
\log \frac{M_{\mathrm{bh}}}{10^{8} M_{\odot}}=-1.14+5.15 \log \frac{v_{\mathrm{c}}}{200 \mathrm{~km} \mathrm{~s}^{-1}} .
$$

Equation (5) is semi-observational in the sense that it depends on an observational relation Equation (3) that connects the mass of the blakchole to the total gravitational mass of the galaxy, and on the theoretical relation Equation (4) that connects the mass of the dark matter halo to the circular velocity of the galaxy.

We also derive a theoretical counter part to Equation (5) by using

$$
\log \frac{M_{\mathrm{bh}}}{10^{8} M_{\odot}}=-0.15+1.33 \log \frac{M_{\mathrm{DM}}}{10^{12} M_{\odot}},
$$

reported in Di Matteo et al. (2003) from cosmological simulations, combined with the theoretical prescription of Ferrarese (2002) derived from Bullock et al. (2001), Equation (4) above, to obtain:

$$
\log \frac{M_{\mathrm{bh}}}{10^{8} M_{\odot}}=0.04+4.43 \log \frac{v_{\mathrm{c}}}{200 \mathrm{~km} \mathrm{~s}^{-1}} .
$$

We overplot Equations (5) and (7) in Figure 1. Most of the 53 galaxies fall between these two relations. The six galaxies, the three leftmost of which we do not include in the fits, that are to the left are all early-types. The slopes in Equations (5) and (7) are similar, but the intercepts are different. This difference could be due to the assumptions made in deriving the two equations. In deriving Equation (5) we assumed that mass of the dark matter halo is equal to the total gravitational mass of the galaxy. This assumption affects the intercept of the resulting relation. For example, if the mass of the dark matter halo is $90 \%$ of the total gravitiatonal mass, a reasonable assumption to make, then the intercept in Equation (5) would increase by 0.07 , not enough to make the intercepts comparable. On the other hand, the reason for the difference could be even more fundamental and have to do with the nature of the simulations from which Equation (6) is derived (Di Matteo et al. 2003) 


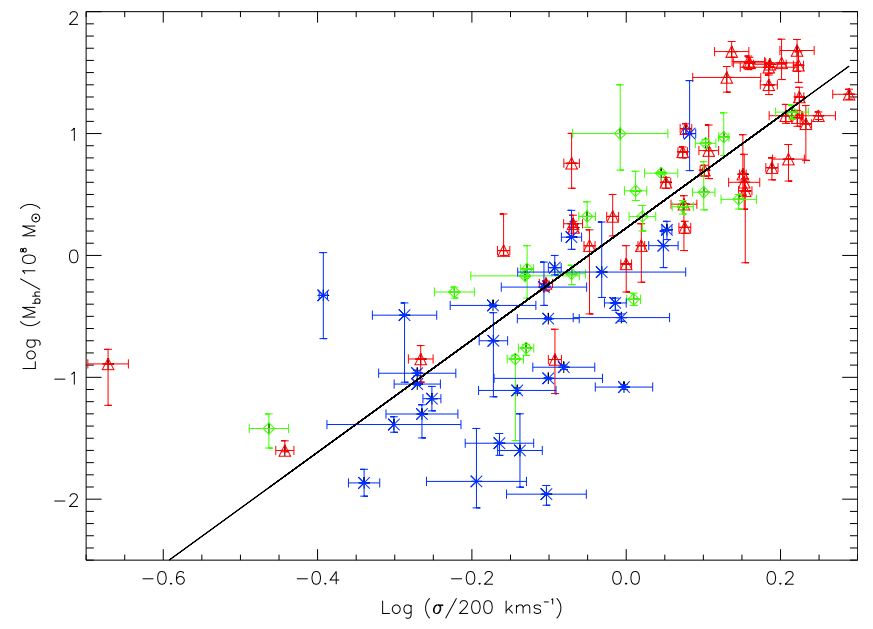

Figure 2. $\log \left(M_{\mathrm{bh}} / 10^{8} M_{\odot}\right)$ vs. $\log \left(\sigma / 200 \mathrm{~km} \mathrm{~s}^{-1}\right)$ for the 89 galaxies in Table 1 that have dynamical black hole mass and host galaxy stellar velocity dispersion measurments. The ellipticals are the red triangles, the lenticulars are the green rhombii, and the spirals are the blue crosses. The solid line is the result of the fit, Equation (8).

\section{2. $M_{\mathrm{bh}}-\sigma$ Relation}

We plot in Figure $2 \log \left(M_{\mathrm{bh}} / 10^{8} M_{\odot}\right)$ versus $\log \left(\sigma / 200 \mathrm{~km} \mathrm{~s}^{-1}\right)$ for the 89 galaxies from Table 1 . The data can be fit by a straight line:

$$
\begin{aligned}
\log \frac{M_{\mathrm{bh}}}{10^{8} M_{\odot}}= & (0.22 \pm 0.06) \\
& +(4.60 \pm 0.31) \log \frac{\sigma}{200 \mathrm{~km} \mathrm{~s}^{-1}}
\end{aligned}
$$

We estimate the intrinsic dispersion to be $\sim 0.50$ dex. The slope and normalization derived here agree, within the errors, with those of Gültenkin et al. (2009a), Hu (2009), Graham (2008), Tremaine et al. (2002), and Merritt \& Ferrarese (2001), however, it is shallower than that reported by Beifiori et al. (2012) and McConnell \& Ma (2013). The relation is also in agreement with theoretical models (Di Matteo et al. 2003).

The amount of intrinsic scatter in our $M_{\mathrm{bh}}-\sigma$ is less than that for the $M_{\mathrm{bh}}-v_{\mathrm{c}}$ above. This is in contrast to the values reported in Volonteri et al. (2011) in which the authors argue that the comparable intrinsic scatter in the $M_{\mathrm{bh}}-\sigma$ and the $M_{\mathrm{bh}}-v_{\mathrm{c}}$ relations indicates that there is a trend of some sort between $M_{\mathrm{bh}}$ and $v_{\mathrm{c}}$. Our sample, consisting of 89 galaxies, is larger than the one used in Gultekin et al. (2009a), 49 galaxies, and Volonteri et al. (2011), 25 galaxies used by Kormendy \& Bender (2011). In contrast, to Figure 1, galaxies in Figure 2 of all morphological types are spread out over roughly the same region.

\subsection{Indirect $M_{\mathrm{bh}}-v_{c}$ ?}

The large scatter in the $M_{\mathrm{bh}}-v_{\mathrm{c}}$ plot (Figure 1) is contrary to what was found in some earlier studies (Ferrarese 2002; Baes et al. 2003). Baes et al. (2003) have calculated the black hole masses indirectly by relying on the $M_{\mathrm{bh}}-\sigma$ relation and then plotted $M_{\mathrm{bh}}$ against $v_{\mathrm{c}}$. They used the $M_{\mathrm{bh}}-\sigma$ relation of Tremaine et al. (2002) with their $v_{\mathrm{c}}-\sigma$ relation to derive a $M_{\mathrm{bh}}-$ $v_{\mathrm{c}}$ relation. It is important to keep in mind that Baes et al. (2003) did not fit $M_{\mathrm{bh}}$ and $v_{\mathrm{c}}$. Our result here corroborates recent work by Beifiori et al. (2012) and Kormendy \& Bender
(2011), but using a larger sample of dynamical black hole masses.

To circumvent biases that could affect analysis relying on small samples, we use the very large sample of Ho (2007) to show that there will be a significant scatter in the $M_{\mathrm{bh}}-v_{\mathrm{c}}$ plots, even when using a $M_{\mathrm{bh}}-\sigma$ relation, which by itself could introduce a relation which is not necessarily there (see our discussion above of Ferrarese (2002) and Baes et al. (2003). Ho (2007) presents $\sigma$ and $v_{\mathrm{c}}$, together with other information, on 792 galaxies to study the $v_{\mathrm{c}}-\sigma$ relation. Given the $\sigma \mathrm{s}$ in Ho (2007), we use our $M_{\mathrm{bh}}-\sigma$ relation, Equation (8), to calculate the corresponding black hole masses for all the 792 galaxies. The errors on $\sigma$ and the uncertainties on the slope and normalization of the $M_{\mathrm{bh}}-\sigma$ relation are propagated in quadrature together with the intrinsic scatter in $M_{\mathrm{bh}}$ at constant $\sigma$ to calculate the uncertainties on the derived $M_{\mathrm{bh}} \mathrm{s}$. We then plot the calculated masses against the corresponding $v_{\mathrm{c}}$ for the 792 objects (Figure 3 ). The green circles are "kinematically normal" galaxies (Ho 2007) with $1 \lesssim \frac{v_{\mathrm{c}}}{\sigma} \lesssim 2$. We also overplot Equation (5), which is based on the observed correlation between $M_{\mathrm{bh}}$ and $M_{\mathrm{DM}}$ (Bandara et al. 2009), and Equation (7), which is based on the theoretical correlation between $M_{\mathrm{bh}}$ and $M_{\text {DM }}$ (Di Matteo et al. 2003). As a visual aid, the horizontal line marks the black hole mass obtained from Equation (8) for $\sigma=100 \mathrm{~km} \mathrm{~s}^{-1}$.

There is a significant amount of scatter with many galaxies occupying a wide swath with $100 \lesssim v_{\mathrm{c}} \lesssim 400 \mathrm{~km} \mathrm{~s}^{-1}$ and $2 \times 10^{6} \lesssim M_{\mathrm{bh}} \lesssim 3 \times 10^{8} \quad M_{\odot} ;$ roughly the same region bounded by Equations (5) and (7). Most of the galaxies in this region are "kinematically normal" (Ho 2007). The majority of galaxies in this region are spirals (Sa to Sc, Figures 3(d)(e)). Moreover, for any given morphological type, the spirals have the highest percentage of occupying this region. However, the region has quite a large scatter, roughly an order of magnitude, in $M_{\mathrm{bh}}$ for a given $v_{\mathrm{c}}$. This makes using it as a kind of a relation between the mass of central black hole and the circular velocity of the host galaxy problematic, or at least not as beneficial as the $M_{\mathrm{bh}}-\sigma$ relation, which is used as probe of the co-evolution, in terms of redshift and morphology, of galaxy spheroids and the SMBH.

Apart from showing that masses of the SMBH are poorly correlated with the circular velocities of their host galaxies, Figure 3 uncovers a few interesting points. There are virtually no galaxies that have high circular velocities but low black hole masses (empty lower right corner), whereas low circular velocity apparently places no constraints on the mass of the black hole (the vertical scatter with $30 \lesssim v_{\mathrm{c}} \lesssim 150 \mathrm{~km} \mathrm{~s}^{-1}$ ). The first part is expected: a high circular velocity is related to a more massive halo, and a more massive halo leads to a deeper potential well, and hence more infalling material that eventually accretes onto the central SMBH.

We address the second part through studying the $M_{\mathrm{bh}}-v_{\mathrm{c}}$ plot, Figure 3(a), by morphological type (Figures 3(b)-(e)). Many elliptical and lenticular galaxies have high black hole masses, $M_{\mathrm{bh}} \gtrsim 10^{7} M_{\odot}$, but with circular velocities as low as $25 \mathrm{~km} \mathrm{~s}^{-1}$ and as high as $500 \mathrm{~km} \mathrm{~s}^{-1}$. They form the horizontal scatter in Figures 3(b) and (c). These galaxies have $100 \lesssim \sigma \lesssim 300 \mathrm{~km} \mathrm{~s}^{-1}$. The horizontal scatter shows that the black hole mass and the circular velocity of the host galaxies are not correlated in any way. However, one must be careful in the interpretation. The circular velocities used here are based on integrated $\mathrm{H}_{\mathrm{I}}$ velocity profiles, which are less than velocities 

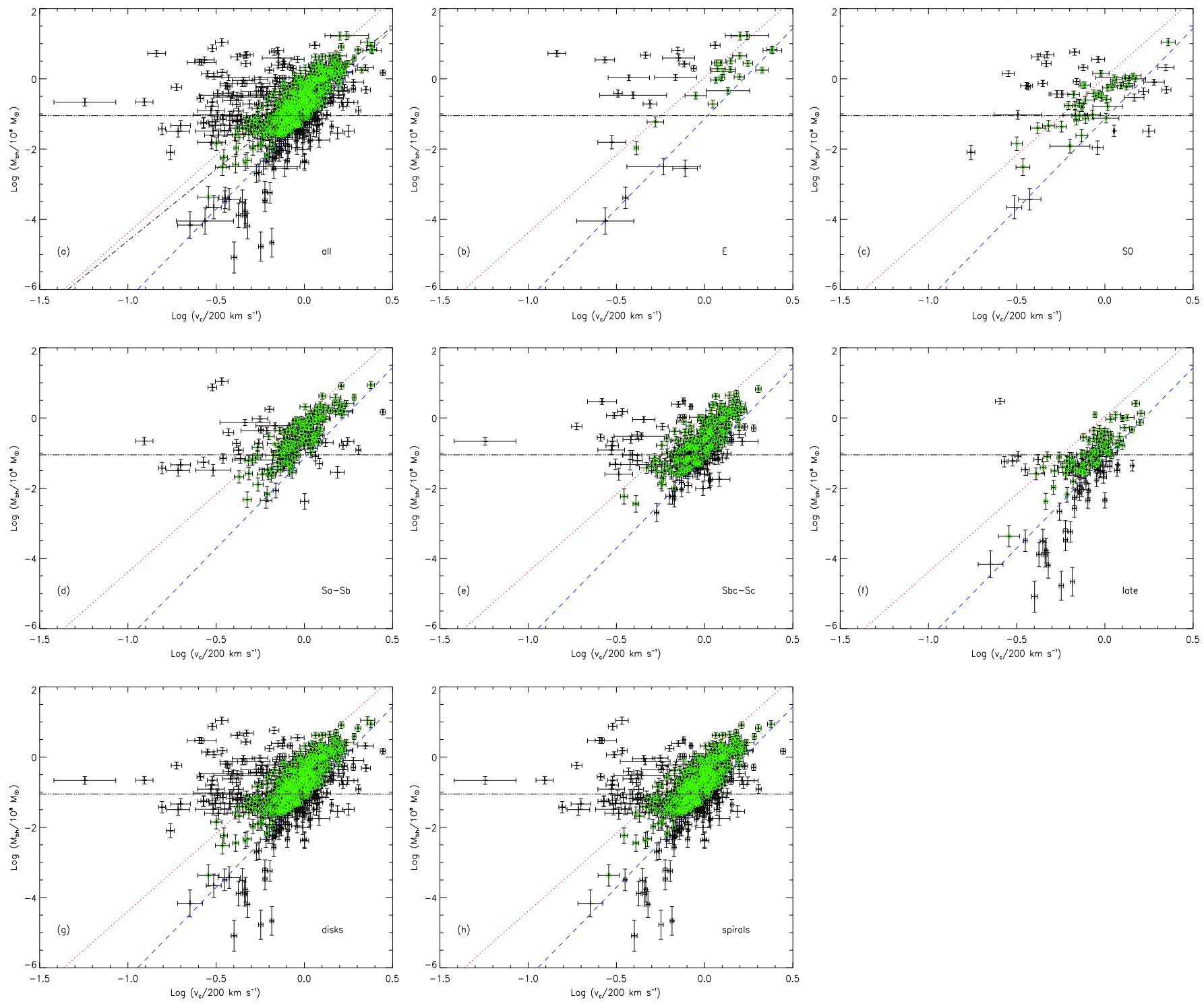

Figure 3. $\log \left(M_{\mathrm{bh}} / 10^{8} M_{\odot}\right)$ vs. $\log \left(v_{\mathrm{c}} / 200 \mathrm{~km} \mathrm{~s}^{-1}\right)$ for the 792 galaxies in the sample of Ho (2007). The black hole masses have been calculated using from the stellar velocity dispersion according to Equation (8). (a): all types, (b): ellipticals, (c): S0 galaxies, (d): Sa and Sb spirals, (e): Sbc and Sc spirals, (f): later than Sc spirals, (g): all disk galaxies, and (h): all spiral galaxies. The blue dashed line is the semitheoretical $M_{\mathrm{bh}}-v_{\mathrm{c}}$ relation Equation (5), and the red dotted line is Equation (7), a theoretical $M_{\mathrm{bh}}-v_{\mathrm{c}}$ relation. Kinematically normal galaxies (see text for definition) are the green circles. The black dotted-dashed line in (a) is the fit to all 616 kinematically normal galaxies. The black horizontal line is the black hole mass calculated from Equation (8) for $\sigma=100 \mathrm{kms} \mathrm{s}^{-1}$.

obtained from optical rotation curves for early type galaxies (see Ho 2007). The discrepancy is almost a factor of 2. This can explain the spread of early type galaxies (morphological index $\lesssim 0)$ to the left in Figures $3(\mathrm{~b})$ and $(\mathrm{c})$, and also in Figure 3(d) (Sa and Sb spirals). One the other hand, the points in the lower part of Figure 3(f) are very late type, bulgeless galaxies whose stellar velocity dispersion is $\lesssim 50 \mathrm{~km} \mathrm{~s}^{-1}$ and most of it is contributed by the rotation of the disk.

It seems that the only galaxies that follow some sort of a correlation are the kinematically normal galaxies. However, the reasoning here seems circular. This could be another manifestation of the "cosmic conspiracy" discussed in Kormendy \& Bender (2011). The kinematically normal galaxies by definition have $1 \lesssim \frac{v_{\mathrm{c}}}{\sigma} \lesssim 2$ with an average $\frac{v_{\mathrm{c}}}{\sigma} \approx \sqrt{2}$. This fact, taken with the $M_{\mathrm{bh}}-\sigma$ relation (Equation (8)), means that $M_{\mathrm{bh}} \propto v_{c}^{4}$. If we fit the 616 kinematically normal galaxies in Figure 3(a), we get:

$$
\begin{aligned}
\log \frac{M_{\mathrm{bh}}}{10^{8} M_{\odot}}= & (-0.55 \pm 0.02) \\
& +(4.07 \pm 0.16) \log \frac{v_{\mathrm{c}}}{200 \mathrm{~km} \mathrm{~s}^{-1}},
\end{aligned}
$$

with no intrinsic dispersion, since it was already included in the calculation of the error on the derived $M_{\mathrm{bh}}$. If this intrinsic scatter is not included, then the intrinsic dispersion in Equation (9) is $\sim 0.9 \mathrm{dex}$. The slope is consistent with that expected from a $M_{\mathrm{bh}}-v_{\mathrm{c}}$ relation via a $M_{\mathrm{bh}}-\sigma$ : the expected slope is $\sim 4$. The intrinsic scatter is significantly less than when fitting using dynamical $M_{\mathrm{bh}}$ as in Equation (2). The intrinsic scatter would disappear if we include the intrinsic dispersion of our $M_{\mathrm{bh}}-\sigma$ relation in the errors of the calculated black hole masses. This expected intrinsic scatter in this case is built in, 
and it behaves like another source of error since it dominates the measurement errors.

\section{CONCLUSIONS}

This paper highlights the importance of the need to use direct values of the quantities being used to study the relation. We attempted here to use dynamical measurements of black hole masses since these measurements are "clean" in the sense that they do not depend on the properties of the host galaxy. Another issue that deserves similar attention is getting similarily "clean" measurements of the circular velocities. Circular velocities depend on the where they are being measured in the galaxy, and the conversion between the circular velocity of the galaxy and the circular velocity of the halo is model dependent. In this study we took the pragmatic approach used by previous authors (e.g., Kormendy \& Bender 2011; Beifiori et al. 2012; Sun 2013) and implicitly assumed that all circular velocities are those of the dark matter halo.

Using this assumption, we found that the correlation between the masses of the SMBHs and the circular velocities is very weak. By extrapolation, we conclude that the correlation between the black holes and the dark matter halo is also weak in the present-day universe. Volonteri et al. (2011) argued the these two components of galaxies could have been more correlated in the past. Black holes and dark matter halos are bound to be correlated somehow. The deep potential wells of massive dark matter halos are needed for the material to be accumulated in the center and form a black hole. The existence of bulgeless spiral galaxies with SMBHs presents a challenge to the no $M_{\mathrm{bh}}-v_{\mathrm{c}}$ correlation. On the other hand, a $M_{\mathrm{bh}}-v_{\mathrm{c}}$ correlation persisting to the present would imply the existence of very massive black holes in the cores of dark matter halos of galaxy clusters (Kormendy \& Bender 2011).

We thank the anonymous referee for helpful comments, and the scientific editor for pertinent suggestions. BMS wishes to thank the Abdus Salam International Center for Theoretical Physics (ICTP) for hospitality, and P. Monaco and P. Salucci for helpful discussions.

\section{REFERENCES}

Atkinson, J. W., Collett, J. L., Marconi, A., et al. 2005, MNRAS, 359, 504 Baes, M., Buyle, P., Hau, G. K. T., \& Dejonghe, H. 2003, MNRAS, 341, L44 Bandara, K., Crampton, D., \& Simard, L. 2009, ApJ, 704, 1135 Barth, A. J., Sarzi, M., Rix, Hans-Walter., et al. 2001, ApJ, 555, 685 Bedregal, A. G., Salamanca-Aragon, A., \& Merrifield, M. R. 2006, MNRAS, 373,1125

Beifiori, A., Sarzi, M., Corsini, E. M., et al. 2009, ApJ, 692, 856

Beifiori, A., Courteau, S., Corsini, E. M., \& Zhu, Y. 2012, MNRAS, 419, 2497

Bender, R., Kormendy, J., Bower, G., et al. 2005, ApJ, 631, 280

Bernardi, M., Alonso, M. V., da Costa, L. N., et al. 2002, AJ, 123, 2990

Bower, G. A., Green, R. F., Bender, R., et al. 2001, ApJ, 550, 75

Bullock, J. S., Kolatt, T. S., Sigad, Y., et al. 2001, MNRAS, 321, 559

Capetti, A., Marconi, A., Macchetto, D., \& Axon, D. 2005, A\&A, 431, 465

Cappellari, M., Verolme, E. K., van der Marel, R. P., et al. 2002, ApJ, 578, 787

Cappellari, M., et al. 2007, arXiv:0709.2861v1

Copin, Y., Cretton, N., \& Emsellem, E. 2004, A\&A, 415, 889

Courteau, S., McDonald, M., Widrow, L. M., \& Holtzman, J. 2007, ApJL, $655, \mathrm{~L} 21$

Cretton, N., \& van den Bosch, F. C. 1999, ApJ, 414, 704

Dalla Bonta, E., Ferrarese, L., Corsini, E. M., et al. 2009, ApJ, 690, 537

Davis, Timothy, A., Alatalo, K., Bureau, M., et al. 2013, MNRAS, 429, 534

Davies, R. I., Thomas, J., Genzel, R., et al. 2006, ApJ, 646, 754

de Fransesco, G., Capetti, A., \& Marconi, A. 2006, A\&A, 460, 439

de Fransesco, G., \& Capetti, A. 2008, A\&A, 479, 355
Devereux, N., et al. 2003, AJ, 125, 1226

Di Matteo, T., Croft, R. A. C., Springel, V., \& Hernquist, L. 2003, ApJ, 593, 69 Di Matteo, T., Colberg, J., Springel, V., \& Hernquist, L. D. 2008, ApJ, 676, 33 Ferrarese, L., Ford, H. C., \& Jaffe, W. 1998, IAUSS, 184, 445

Ferrarese, L., \& Ford, H. C. 1999, ApJ, 515, 583

Ferrarese, L., \& Merritt, D. 2000, ApJL, 539, L9

Ferrarese, L. 2002, ApJ, 578, 90

Ferrarese, L., \& Ford, H. C. 2005, SSRv, 116, 523

Sijacki, Garcia-Rissmann, A., Vega, L. R., Asari, N. V., et al. 2005, MNRAS, 359,765

Gebhardt, K., Bender, R., Bower, G., et al. 2000, ApJ, 539, L13

Gebhardt, K., Lauer, T. R., Kormendy, J., et al. 2001, AJ, 122, 2469

Gebhardt, K., Richstone, D., Tremaine, S., et al. 2003, ApJ, 583, 92

Ghez, A. M., Salim, S., Weinberg, N. N., et al. 2008, ApJ, 689, 1044 Gould, A. 2013, arXiv:1303.0834G

Graham, Alister, W., Erwin, Peter, Caon, N., \& Trujillo, I. 2001, ApJ, 563L, 11

Graham, A., \& Driver, S. 2007, ApJ, 655, 77

Graham, A. 2008, PASA, 25, 167

Greene, J. E., \& Ho, L. C. 2005, ApJ, 630, 122

Greenhill, L. J. 2003, ApJL, 590, L62

Greenhill, L. J., Moran, J. M., \& Herrnstein, J. R. 1997, ApJ, 481, 23

Gültekin, K., Richstone, D. O., Gebhardt, K., et al. 2009a, ApJ, 698, 198

Gültekin, K., Richstone, Douglas, O., et al. 2009b, ApJ, 695, 1577

Haehnelt, M., \& Kauffmann, G. 2000, MNRAS, 318, L35

Harms, R. J., Ford, H. C., Tsvetanov, Z. I., et al. 1994, ApJ, 435, L35

Herrnstein, J. R., Moran, J. M., Greenhill, L. J., et al. 1999, Nature, 400, 539

Hicks, E. K. S., \& Malkan, M. S. 2008, ApJS, 174, 31

Ho, L. C. 2007 , ApJ, 668, 94

Ho, L. C., Darling, J., \& Greene, J. E. 2008, ApJ, 681, 128

Hopkins, P. F., Hernquist, L., Cox, T. J., Robertson, B., \& Krause, E. 2007, ApJ, 669, 45

Houghton, R. C. W., Magorrian, J., Sarzi, M., et al. 2006, MNRAS, 367, 2

Hu, J. 2008, MNRAS, 386, 2242

Hu, J. 2009, arXiv:0908.2028v1

Kaspi, S., Smith, P. S., Netzer, H., et al. 2000, ApJ, 533, 631

Kauffmann, G., \& Haehnelt, M. 2000, MNRAS, 311, 567

Kondratko, P. T., Greenhill, L. J., \& Moran, J. M. 2008, ApJ, 678, 87

Kormendy, J. 1988, ApJ, 335, 40

Kormendy, J., Bender, R., Magorrian, J., et al. 1996, AAS, 28, 1423

Kormendy, J., \& Bender, R. 2011, Natur, 469, 377

Krajnovic, D., McDermid, R. M., Cappellari, M., \& Davies, R. L. 2009, MNRAS, 399, 1839

Kronawitter, A., Saglia, R. P., Gerhard, O., \& Bender, R. 2000, A\&AS, 144, 53

Kuo, C. Y., Braatz, J. A., Condon, J. J., et al. 2011, ApJ, 727, 20

Lodato, G., \& Bertin, G. 2003, A\&A, 408, 1015

Maciejewski, W., \& Binney, J. 2001, MNRAS, 323, 831

Macchetto, F., Marconi, A., Axon, D. J., et al. 1997, ApJ, 489, 579

Markwardt, C. B. 2009, ASPC, 411, 251

McConnell, N. J., Ma, C., Murphy, J. D., et al. 2012, ApJ, 756, 179

McConnell, N. J., \& Ma, C. 2013, ApJ, 764, 184

Merritt, D., \& Ferrarese, L. 2001, ApJ, 547, 140

Nelson, C. H., et al. 2004, ApJ, 615, 652

Neistein, E., et al. 1999, AJ, 117, 2666

Neumayer, N., et al. 2007, ApJ, 671, 1329

Nowak, N. 2007, MNRAS, 379, 909

Nowak, N. 2008, MNRAS, 391, 1629

Onken, C. A., Ferrarese, L., Merritt, D., et al. 2004, ApJ, 615, 645

Onken, C. A., Valluri, M., Peterson, B. M., et al. 2007, ApJ, 670, 105

Palunas, P., \& Williams, T. B. 2000, AJ, 120, 2884

Pastorini, G., Marconi, A., Capetti, A., et al. 2007, A\&A, 469, 405

Paturel, G., Theureau, G., Bottinelli, L., et al. 2003, A\&A, 412, 57

Peterson, B. M., Ferrarese, L., Gilbert, K. M., et al. 2004, ApJ, 613, 682

Pizzella, A., Corsini, E. M., Dalla Bontà, E., et al. 2005, ApJ, 631, 785

Press, W. H., Teukolsky, S. A., Vetteriing, W. T., \& Flannery, B. P. 1992,

Numerical Recipes in FORTRAN: The Art of Scientific Computing (2nd ed.; Cambridge: Cambridge Univ. Press)

Prugniel, P., Maubon, G., \& Simien, F. 2001, A\&A, 366, 68

Richstone, D., Ajhar, E. A., Bender, R., et al. 1998, Natur, 395, 14

Richstone, D., et al. 2004, arXiv:astro-ph/0403257v1

Rusli, S. P., Thomas, J., Erwin, P., et al. 2011, MNRAS, 410, 1223

Sabra, Bassem M., Abi Akl, M., \& Chahine, G. 2008, IAUS, 245, 257

Sarzi, M., Rix, H.-W., Shields, J. C., et al. 2001, ApJ, 550, 65

Shapiro, K. L., Cappellari, M., de Zeeuw, T., et al. 2006, MNRAS, 370, 559

Sun, A. 2013, ApJ, 778, 47

Tadhunter, C., Marconi, A., Axon, D., et al. 2003, MNRAS, 342, 861

Tilak, A., Greenhill, L. J., Done, C., \& Madejski, G. 2008, ApJ, 678, 701 
Tremaine, S., Gebhardt, K., Bender, R., et al. 2002, ApJ, 574, 740

Williams, M. J., Bureau, M., \& Cappellari, M. 2010, MNRAS, 409, 1330

Wold, M., Lacy, M., Käufl, H. U., \& Siebenmorgen, R. 2006, A\&A, 460, 449

Valluri, M., Merritt, D., \& Emsellem, E. 2004, ApJ, 602, 66

van den Bosch, R. C. E., Gebhardt, K., Gültekin, K., et al. 2012, Nature, 491,729 van der Marel, R. P., \& van den Bosch, F. C. 1998, AJ, 116, 2220

Verolme, E. K., Cappellari, M., Copin, Y., et al. 2002, MNRAS, 335, 517

Volonteri, M., Natarjan, P., \& Gultekin, K. 2011, ApJ, 737, 50

Yamauchi, A., Nakai, N., Sato, N., \& Diamond, P. 2004, PASJ, 56, 605

Zasov, A. V., Petrochenko, L. N., \& Cherepshchuk, A. M. 2005, ARep, 49,362 